\title{
SISTEMAS DE PREPARO DO SOLO E CULTURAS DE COBERTURA NA PRODUÇÃO ORGÂNICA DE FEIJÃO E MILHO. II - ATRIBUTOS BIOLÓGICOS DO SOLO ${ }^{(1)}$
}

\author{
Eurâimi de Queiroz Cunha ${ }^{(2)}$, Luís Fernando Stone ${ }^{(3)}$, Enderson \\ Petrônio de Brito Ferreira ${ }^{(3)}$, Agostinho Dirceu Didonet ${ }^{(3)}$, José \\ Aloísio Alves Moreira ${ }^{(3)}$ \& Wilson Mozena Leandro ${ }^{(4)}$
}

\begin{abstract}
RESUMO
Há necessidade de se avaliar a contribuição de culturas de cobertura e do seu manejo na manutenção da qualidade biológica do solo em áreas sob produção orgânica. Este trabalho objetivou determinar a influência das plantas de cobertura crotalária (Crotalaria juncea), guandu (Cajanus cajan (L.) Millsp), mucuna-preta (Mucuna aterrima), sorgo-vassoura (Sorghum technicum) e pousio nos atributos biológicos de solo cultivado com feijão e milho orgânicos, sob semeadura direta (SD) e preparo convencional (PC). O trabalho foi conduzido em Santo Antônio de Goiás-GO, em Latossolo Vermelho distrófico, no delineamento de blocos ao acaso, com quatro repetições. Em novembro de 2003 foram instalados quatro experimentos, dois em SD e dois em PC, sendo um com feijão e outro com milho em cada sistema. Amostragens de solo das parcelas e de uma mata próxima aos experimentos foram realizadas em novembro de 2007 , nas camadas de $0,00-0,10$ e 0,10-0,20 m, para determinação do teor de C orgânico total (COT), carbono da biomassa microbiana (CBM), respiração basal do solo (RBS), quociente metabólico $\left(q \mathrm{CO}_{2}\right)$ e quociente microbiano $(q \mathrm{MIC})$. As principais alterações nos atributos biológicos com o uso agrícola ocorreram na camada superficial, onde, de maneira geral, os valores de CBM foram menores que no solo sob mata, sendo esse fato mais pronunciado nas áreas sob $\mathrm{PC} . \quad \mathrm{O} q \mathrm{CO}_{2}$ mostrou-se sensivel às alterações decorrentes do preparo do solo, apresentando valores mais favoráveis na camada superficial do solo sob SD.
\end{abstract}

Termos de indexação: C orgânico do solo, carbono da biomassa microbiana, respiração basal do solo, quociente metabólico, quociente microbiano.

\footnotetext{
(1) Parte da Tese de Doutorado do primeiro autor (projeto financiado pelo CNPq). Recebido para publicação em março de 2010 e aprovado em dezembro de 2010.

(2) Engenheiro Agrônomo, Doutorando em Agronomia pela Universidade Federal de Goiás - UFG. Caixa Postal 131, CEP 74001970 Goiânia (GO). E-mail: euraimi@yahoo.com.br

(3) Pesquisador, Embrapa Arroz e Feijão, GO 462, km 12, Caixa Postal 179, CEP 75375-000 Santo Antônio de Goiás (GO). Emails: stone@cnpaf.embrapa.br; enderson@cnpaf.embrapa.br; didonet@cnpaf.embrapa.br; jaloisio@cnpaf.embrapa.br

(4) Professor, Escola de Agronomia e Engenharia de Alimentos, Universidade Federal de Goiás - UFG. Bolsista do CNPq. E-mail: wilson.mozena@pq.cnpq.br
} 


\title{
SUMMARY: SOIL TILLAGE SYSTEMS AND COVER CROPS IN ORGANIC PRODUCTION OF COMMON BEAN AND CORN. II - SOIL BIOLOGICAL PROPERTIES
}

\begin{abstract}
The influence of cover crops and their management on the maintenance of soil biological quality in areas under organic production should be better understood. This study aimed to determine the influence of the cover crops sunn hemp (Crotalaria juncea), pigeon pea (Cajanus cajan (L.) Millsp), velvet bean (Mucuna aterrima), sorghum (Sorgum technicum), and fallow on biological properties of soil under organic common bean and corn, in no-tillage (NT) and conventional tillage (CT) systems. The study was conducted in Santo Antônio de Goiás-GO, on an Oxisol, in a randomized block design, with four replications. Four experiments were installed in November 2003, two of them under NT and the other two in CT. In each soil management system, one experiment was carried out with common bean and another with corn. Samples were taken from the layers 0.00-0.10 and 0.10-0.20 $\mathrm{m}$ in November 2007, in the plots and in a neighboring native forest, to determine the soil organic carbon content (SOC), microbial biomass carbon (MBC), soil basal respiration ( $\mathrm{SBR}$ ), metabolic quotient (q $\left.\mathrm{CO}_{2}\right)$, and microbial quotient (qMIC). The main changes in biological properties of soil under agricultural use occurred in the surface layer, where, in general, the MBC values were lower than in forest soil, a difference that was more pronounced in CT areas. The $\mathrm{qCO}_{2}$ was sensitive to changes resulting from soil tillage, with lowest values in the surface layer of NT soil.
\end{abstract}

Index terms: soil organic carbon, microbial biomass carbon, soil basal respiration, metabolic quotient, microbial quotient.

\section{INTRODUÇÃO}

Estudos visando identificar indicadores de alterações biológicas em áreas sob cultivo orgânico são importantes para determinar a sustentabilidade desse sistema de cultivo.

A qualidade do solo é fortemente influenciada por processos mediados por microrganismos e, como ela pode estar relacionada à sua diversidade, é provável que a estrutura da comunidade microbiana tenha o potencial para servir como uma indicação precoce da degradação ou melhoria da qualidade do solo (Islam \& Weil, 2000b). Esses autores constataram que a biomassa microbiana (BMS) e a respiração basal do solo por unidade de BMS mostraram-se mais promissores para inclusão em um índice de qualidade de solo, com base em amostras de solo de sistemas de manejo contrastantes.

Os atributos biológicos do solo têm a capacidade de medir o nível de desequilíbrio ao qual um determinado ambiente está sujeito, sendo úteis para determinar os efeitos positivos e negativos sobre a qualidade do solo e a sustentabilidade das práticas agrícolas. Atributos como a BMS fornecem informações que servirão de subsídios para avaliação da qualidade do solo (Alvarez et al., 1995).

A BMS desempenha papel fundamental na produtividade e na manutenção dos ecossistemas, pois atua como um catalisador das importantes transformações químicas no solo e constitui um reservatório de nutrientes disponíveis às plantas, por pertencer ao componente lábil da matéria orgânica do solo e possuir atividade influenciada pelas condições bióticas e abióticas. O seu acompanhamento reflete possíveis modificações no solo, sendo considerada uma boa indicadora das alterações resultantes do manejo (D'Andrea et al., 2002; Souza et al., 2006). Como ela constitui a maior parte da fração ativa da matéria orgânica, ela é mais sensível que o resultado quantitativo do C orgânico e do $\mathrm{N}$ total para aferir alterações na matéria orgânica causadas pelo manejo do solo e pelas práticas de cultivo (Santos \& Camargo, 1999). Há tendência de a BMS ser maior em camadas mais superficiais, pela maior disponibilidade de matéria orgânica, água e nutrientes (Silva \& Mendonça, 2007).

Sistemas de manejo que condicionam aumentos em atributos biológicos associados à biomassa microbiana proporcionam incremento na ciclagem de nutrientes e energia no sistema solo. A inclusão de leguminosas em sistemas agrícolas é uma estratégia que deve ser avaliada em relação ao seu efeito nos estoques de matéria orgânica do solo. É provável que o aumento da produtividade das culturas comerciais em sucessão a leguminosas também ocasione incremento na adição de resíduos não colhidos ao solo, favorecendo o acúmulo de matéria orgânica (Amado et al., 2001). Esses autores verificaram que a utilização de mucuna (Stizolobium cinereum) no plantio direto do milho promoveu aumento nos estoques de C orgânico e N total do solo. Souza et al. (2006), em estudo realizado com sete sistemas de culturas, observaram que aqueles com maior produção de biomassa vegetal e acúmulo de resíduos na superfície apresentaram os maiores valores para a biomassa microbiana na camada de até $0,05 \mathrm{~m}$. 
Comparando o efeito de diferentes sistemas de manejo sobre a qualidade do solo, tendo o manejo conservacionista como padrão de comparação, relatos têm mostrado que as práticas que sustentam a matéria orgânica melhoram a qualidade do solo (Islam \& Weil, 2000b). A biomassa microbiana, segundo Vasconcellos et al. (1999), imobiliza mais carbono quando resíduos são deixados na superfície, havendo, com isso, tendência linear de crescimento da BMS em áreas sob semeadura direta. Realmente, Santos et al. (2007) verificaram que o valor da BMS foi maior no sistema de semeadura direta em relação ao preparo convencional do solo. Mendes et al. (2003) e Silva et al. (2007) constataram maiores valores de carbono da biomassa microbiana (CBM) em solo sob semeadura direta, o que indica que esse manejo favorece a atividade biológica do solo. D'Andréa et al. (2002) observaram decréscimo do CBM com o uso do Cerrado nativo para pastagem ou sistemas agrícolas sob semeadura direta e preparo convencional, com a maior redução verificada no plantio convencional de longa duração, e a menor, na pastagem.

A respiração basal (RBS), que é a liberação de C$\mathrm{CO}_{2}$ de amostras de solo, é um indicador da atividade heterotrófica da biomassa, sendo a quantidade de C liberado indicativo do C lábil ou prontamente metabolizável do solo (Jenkinson \& Powlson, 1976). A relação entre a RBS e o CBM é denominada de quociente metabólico $\left(q \mathrm{CO}_{2}\right)$, e a relação entre o $\mathrm{C}$ da biomassa microbiana (CBM) e o C orgânico do solo, quociente microbiano ( $q \mathrm{MIC}$ ) (Anderson \& Domsch, 1993). Esses atributos biológicos também podem ser utilizados para avaliação da qualidade do solo, pois, sob estresse, a BMS apresenta $q \mathrm{CO}_{2}$ e RBS mais altos, indicando maior consumo de energia. Devem, contudo, ser comparados em um mesmo solo, uma vez que outros fatores, além do estresse, podem afetar a BMS (Moreira \& Siqueira, 2006). Portanto, devem ser buscados sistemas que promovam menores $q \mathrm{CO}_{2}$, pois, neles, a BMS está em equilíbrio, com menores perdas de $\mathrm{CO}_{2}$ pela respiração; com isso, maior é a incorporação de $\mathrm{C}$ à BMS.

Costa et al. (2006) observaram maior CBM em área de Cerrado nativo em relação a áreas sob preparo convencional e semeadura direta. Observaram, ainda, maior RBS em áreas de Cerrado nativo e sob semeadura direta com milho (maior adição de restos culturais com maior relação $\mathrm{C} / \mathrm{N}$ ) em relação àquela sob preparo convencional com milho. Araújo et al. (2007) também constataram maior CBM e RBS em área de Cerrado nativo em comparação ao cultivo convencional com milho. Conceição et al. (2005), entretanto, não observaram diferenças significativas nos valores de CBM entre diferentes sistemas de preparo do solo e de culturas antecessoras ao milho. D’Andréa et al. (2002), por sua vez, não encontraram diferenças nos valores de RBS entre Cerrado nativo, pastagem e sistemas agrícolas sob preparo convencional e semeadura direta.
Tendo em vista não haver ainda consenso sobre o número e o tipo dos atributos biológicos necessários para estimar a qualidade do solo (Bremer \& Ellert, 2004), deve-se ter cautela quanto ao seu uso. Assim, por exemplo, alta atividade microbiana não é necessariamente indicativo de melhoria na qualidade do solo, podendo inclusive ser considerada um fator negativo, em virtude de acelerar a decomposição de resíduos orgânicos e, portanto, diminuir o tempo de residência da matéria orgânica do solo (Araújo et al., 2007).

O efeito de plantas de cobertura e de sistemas de preparo no acúmulo de matéria orgânica no solo e na melhoria de seus atributos biológicos deve ser quantificado regionalmente e para cada sistema produtivo, uma vez que depende da textura e mineralogia do solo, do relevo e das condições de temperatura e umidade. Assim, considerando que existe pouca informação sobre indicadores de qualidade do solo em sistema de produção orgânica, o presente trabalho teve como objetivo determinar a influência de plantas de cobertura nos atributos biológicos de solo cultivado com feijão e milho orgânicos, sob semeadura direta e preparo convencional, na região do Cerrado.

\section{MATERIAL E MÉTODOS}

\section{Caracterização do local, delineamento experimental e tratamentos}

O trabalho foi conduzido na Fazenda Capivara, da Embrapa Arroz e Feijão, localizada no município de Santo Antônio de Goiás-GO. O solo do local é um Latossolo Vermelho distrófico (Embrapa, 2006), de textura franco-argilosa, com $410 \mathrm{~g} \mathrm{~kg}^{-1}$ de areia, $270 \mathrm{~g} \mathrm{~kg}^{-1}$ de silte e $320 \mathrm{~g} \mathrm{~kg}^{-1}$ de argila, na camada de $0,00-0,20 \mathrm{~m}$. A vegetação original da área experimental era do tipo Cerradão (Sousa-Silva \& Camargo, 2008) e vinha sendo cultivada no sistema convencional de preparo do solo (gradagens aradora e niveladora) com a rotação milho e soja.

Em novembro de 2003 foram instalados quatro experimentos, que estão sendo conduzidos segundo os preceitos da produção orgânica. Dois experimentos foram conduzidos em semeadura direta, e nos outros dois foi feito o preparo convencional do solo com grades aradora e niveladora operando até $0,10-0,15 \mathrm{~m}$ de profundidade. Em cada sistema de preparo do solo, foi conduzido um experimento com a cultura do milho e outro com a do feijão das águas. Em todos os experimentos foram comparadas, no delineamento de blocos ao acaso, com quatro repetições, as culturas de cobertura: crotalária (Crotalaria juncea), guandu (Cajanus cajan (L.) Millsp), mucuna-preta (Mucuna aterrima), sorgo-vassoura (Sorghum technicum) e pousio (vegetação espontânea). Mais detalhes são apresentados em Cunha et al. (2011). 


\section{Amostragem e determinações}

Em novembro de 2007 foi realizada amostragem de solo para análise biológica nas camadas de 0,00 0,10 e 0,10-0,20 m. Foi também amostrado o solo de uma mata próxima ao experimento (Cerradão), representativa da vegetação original. Foram retiradas seis amostras por parcela, que foram acondicionadas em sacos plásticos e levadas imediatamente ao laboratório. As amostras foram peneiradas em malha de $2 \mathrm{~mm}$ e a umidade ajustada para $80 \%$ da capacidade de campo, sendo acondicionadas em sacos plásticos e mantidas sob refrigeração $\left(4^{\circ} \mathrm{C}\right)$ até a realização das análises de biomassa e respiração. Os atributos biológicos avaliados foram $\mathrm{C}$ da biomassa microbiana (CBM), determinado pelo método da fumigação-extração proposto por Vance et al. (1987), na qual a biomassa microbiana é estimada com base na diferença do fluxo de $\mathrm{CO}_{2}$ de três amostras fumigadas com clorofórmio por parcela e três não fumigadas; respiração basal do solo (RBS), determinada pela quantificação do $\mathrm{CO}_{2}$ liberado a partir de $20 \mathrm{~g}$ de solo durante a incubação do solo por sete dias em sistema fechado e capturado em frascos com KOH (Jenkinson \& Powlson, 1976); e quocientes metabólico $\left(q \mathrm{CO}_{2}\right)$, obtido pela relação entre RBS e $\mathrm{CBM}$, e microbiano ( $q \mathrm{MIC}$ ), obtido pela relação entre CBM e C orgânico total do solo (COT) (Anderson \& Domsch, 1993). O COT foi determinado pelo método de Walkley \& Black (Embrapa, 1997), que consiste na oxidação do $\mathrm{C}$ orgânico do solo pelo $\mathrm{Cr}^{6+}$ na presença de $\mathrm{H}_{2} \mathrm{SO}_{4}$ concentrado, na qual o excesso de $\mathrm{Cr}^{6+}$ é titulado com $\mathrm{Fe}^{2+}$.

\section{Análise estatística}

Os dados foram submetidos à análise de variância por camada, utilizando-se o procedimento GLM do programa estatístico SAS (SAS, 1999), sendo as médias comparadas pelo teste de Tukey a $5 \%$. As médias dos tratamentos foram comparadas com as obtidas no solo da mata nativa, pelo teste de Dunnett a $5 \%$. Foram estimadas correlações de Pearson entre as variáveis estudadas, considerando-se, conjuntamente, as duas camadas amostradas.

\section{RESULTADOS E DISCUSSÃO}

\section{Carbono orgânico total do solo (COT)}

Nos experimentos com as culturas do feijoeiro e do milho não foram observadas diferenças significativas entre as plantas de cobertura quanto ao COT, tanto na camada de $0,00-0,10 \mathrm{~m}$ como na de $0,10-0,20 \mathrm{~m}$, seja sob preparo convencional (Quadro 1), seja sob semeadura direta (Quadro 1).

Nascimento et al. (2005), após três anos de cultivo, comparando 12 leguminosas e uma parcela com vegetação espontânea, também não verificaram influência das plantas de cobertura no COT. Almeida et al. (2008), comparando por três anos áreas sob pousio, crotalária (Crotalaria juncea L.), guandu (Cajanus cajan (L.) Millsp), mucuna-preta (Mucuna aterrima, sinonímia (Stizolobium aterrimum Piper $\&$ Tracy) e milheto (Pennisetum americanum L.), e Sousa Neto et al. (2008), comparando por quatro anos crotalária, milheto e lablabe (Dolichus lablab), não verificaram influência das plantas de cobertura no teor de matéria orgânica do solo. Nas duas camadas, em todos os experimentos, o COT sob mata foi maior do que sob as plantas de cobertura, provavelmente pelo grande aporte de resíduos orgânicos, não revolvimento do solo e reduzida erosão hídrica pela maior cobertura do solo pela liteira, concordando com os resultados de Albuquerque et al. (2005), Jakelaitis et al. (2008) e Pôrto et al. (2009). De acordo com Jakelaitis et al. (2008), a diminuição no COT nos solos sob cultivo pode ser devida também ao aumento no consumo do $\mathrm{C}$ prontamente disponível pela biomassa microbiana e, ainda, pelo manejo adotado.

Tomando a mata como referência, observou-se redução similar nos valores médios de COT para os experimentos sob preparo convencional, 45,4 e $38,5 \%$, e para os sob semeadura direta, 44,4 e 40,4\%, respectivamente com as culturas do feijoeiro e do milho.

\section{Carbono da biomassa microbiana (CBM)}

Foram observadas diferenças significativas entre as plantas de cobertura, quanto ao teor de CBM do solo, apenas na camada de 0,00-0,10 $\mathrm{m}$ dos experimentos com o feijoeiro. No experimento sob preparo convencional (Quadro 1), o guandu propiciou maiores valores desse atributo em relação às demais plantas de cobertura, com exceção da mucuna. Sob semeadura direta (Quadro 1), o teor de CBM foi maior sob guandu e mucuna em relação à crotalária e ao pousio. Silva et al. (2007), comparando diversas culturas usadas como cobertura do solo para o feijoeiro irrigado, nesses dois sistemas de preparo do solo, não encontraram diferença quanto a esse atributo.

Houve diferenças significativas entre as plantas de cobertura e a mata, quanto ao teor de CBM, apenas na camada superficial do solo, onde, de maneira geral, esse atributo no solo sob mata foi maior do que sob as plantas de cobertura (Quadro 1), especialmente sob preparo convencional, concordando com os resultados de D'Andréa et al. (2002), Costa et al. (2006), Silva et al. (2007) e Pôrto et al. (2009). Nessa camada são mais intensos os processos de transformação da matéria orgânica pelos microrganismos do solo. Tomando a mata como referência, observou-se redução nos valores médios de CBM da ordem de 47,5 e 46,4\% para os experimentos sob preparo convencional e de 25,6 e $34,6 \%$ para os sob semeadura direta, respectivamente com as culturas do feijoeiro e do milho. Verificou-se ainda que essa diferença entre os 
Quadro 1. Carbono orgânico total (COT), carbono da biomassa microbiana (CBM), respiração basal (RBS), quociente metabólico $\left(q \mathrm{CO}_{2}\right)$ e quociente microbiano( $\left.q \mathrm{MIC}\right)$ de acordo com as culturas de cobertura e camadas estudadas, no experimento sob preparo convencional com a cultura do feijoeiro, no experimento sob semeadura direta com a cultura do feijoeiro, no experimento sob preparo convencional com a cultura do milho e no experimento sob semeadura direta com a cultura do milho

\begin{tabular}{|c|c|c|c|c|c|c|c|c|c|c|}
\hline \multirow{3}{*}{ Tratamento } & \multicolumn{10}{|c|}{ Profundidade (m) } \\
\hline & $0,00-0,10$ & $0,10-0,20$ & $0,00-0,10$ & $0,10-0,20$ & $0,00-0,10$ & $0,10-0,20$ & $0,00-0,10$ & $0,10-0,20$ & $0,00-0,10$ & $0,10-0,20$ \\
\hline & \multicolumn{2}{|r|}{ COT } & \multicolumn{2}{|c|}{ CBM } & \multicolumn{2}{|c|}{ RBS } & \multicolumn{2}{|c|}{$q \mathrm{CO}_{2}$} & \multicolumn{2}{|r|}{$q \mathrm{MIC}$} \\
\hline & \multicolumn{2}{|c|}{$\mathrm{g} \mathrm{kg}^{-1}$} & \multicolumn{2}{|c|}{ — $\mathrm{mg} \mathrm{kg}^{-1} \mathrm{de} \mathrm{C}-$} & \multicolumn{2}{|c|}{$m g \mathrm{~kg}^{-1} \mathrm{~h}^{-1}$ de C-CO} & \multicolumn{2}{|c|}{$\mathrm{mg} \mathrm{kg}^{-1} \mathrm{~h}^{-1}$ de C-CO ${ }_{2}$ do $\mathrm{CBN}$} & \multicolumn{2}{|r|}{$-\%$} \\
\hline & \multicolumn{10}{|c|}{ Preparo convencional com a cultura do feijoeiro } \\
\hline $\begin{array}{l}\text { Pousio } \\
\text { Crotalária } \\
\text { Guandu } \\
\text { Mucuna } \\
\text { Sorgo }\end{array}$ & $\begin{array}{l}9,15 a^{*} \\
9,56 a^{*} \\
9,13 a^{*} \\
9,12 a^{*} \\
9,54 a^{*}\end{array}$ & $\begin{array}{l}8,32 \mathrm{a}^{*} \\
8,57 \mathrm{a}^{*} \\
8,71 \mathrm{a}^{*} \\
8,38 \mathrm{a}^{*} \\
8,27 \mathrm{a}^{*}\end{array}$ & $\begin{array}{l}178,0 \mathrm{c}^{*} \\
195,5 \mathrm{bc} * \\
293,8 \mathrm{a} * \\
246,5 \mathrm{ab} * \\
174,0 \mathrm{c}^{*}\end{array}$ & $\begin{array}{l}215,9 \mathrm{a} \\
226,0 \mathrm{a} \\
245,5 \mathrm{a} \\
233,2 \mathrm{a} \\
230,1 \mathrm{a}\end{array}$ & $\begin{array}{l}0,64 \mathrm{a} \\
0,59 \mathrm{a} \\
0,81 \mathrm{a} \\
0,64 \mathrm{a} \\
0,58 \mathrm{a}\end{array}$ & $\begin{array}{c}0,23 \mathrm{a} \\
0,23 \mathrm{a} \\
0,29 \mathrm{a} \\
0,25 \mathrm{a} \\
0,27 \mathrm{a}\end{array}$ & $\begin{array}{l}3,59 \mathrm{a} \\
3,06 \mathrm{a} \\
2,76 \mathrm{a} \\
2,67 \mathrm{a} \\
3,34 \mathrm{a}\end{array}$ & $\begin{array}{l}1,15 \mathrm{a} \\
1,01 \mathrm{a} \\
1,19 \mathrm{a} \\
1,07 \mathrm{a} \\
1,32 \mathrm{a}\end{array}$ & $\begin{array}{l}1,96 \mathrm{bc} \\
2,04 \mathrm{bc} \\
3,22 \mathrm{a}^{*} \\
2,70 \mathrm{ab} \\
1,85 \mathrm{c}\end{array}$ & $\begin{array}{l}2,60 a^{*} \\
2,64 a^{*} \\
2,83 a * \\
2,79 a * \\
2,81 a^{*}\end{array}$ \\
\hline \multirow[t]{2}{*}{$\begin{array}{l}\text { CV (\%) } \\
\text { Mata }\end{array}$} & $\begin{array}{c}6,4 \\
17,03\end{array}$ & $\begin{array}{c}4,5 \\
14,53\end{array}$ & $\begin{array}{r}12,8 \\
414,6\end{array}$ & $\begin{array}{r}18,0 \\
214,0\end{array}$ & $\begin{array}{c}30,9 \\
0,88\end{array}$ & $\begin{array}{c}18,9 \\
0,24\end{array}$ & $\begin{array}{c}26,2 \\
2,10\end{array}$ & $\begin{array}{c}29,1 \\
1,13\end{array}$ & $\begin{array}{c}14,4 \\
2,44\end{array}$ & $\begin{array}{l}21,7 \\
1,49\end{array}$ \\
\hline & \multicolumn{10}{|c|}{ Semeadura direta com a cultura do feijoeiro } \\
\hline $\begin{array}{l}\text { Pousio } \\
\text { Crotalária } \\
\text { Guandu } \\
\text { Mucuna } \\
\text { Sorgo }\end{array}$ & $\begin{array}{l}9,09 a^{*} \\
9,46 a^{*} \\
9,34 a^{*} \\
9,48 a^{*} \\
9,96 a^{*}\end{array}$ & $\begin{array}{l}8,59 a^{*} \\
8,59 a^{*} \\
8,35 a^{*} \\
8,56 a^{*} \\
8,52 a^{*}\end{array}$ & $\begin{array}{l}232,7 \mathrm{bc} * \\
219,4 c^{*} \\
398,0 \mathrm{a} \\
356,3 \mathrm{a} \\
335,1 \mathrm{ab}\end{array}$ & $\begin{array}{l}198,2 \mathrm{a} \\
223,0 \mathrm{a} \\
219,8 \mathrm{a} \\
173,1 \mathrm{a} \\
177,0 \mathrm{a}\end{array}$ & $\begin{array}{l}0,32 a^{*} \\
0,29 a^{*} \\
0,21 a^{*} \\
0,21 a^{*} \\
0,29 a^{*}\end{array}$ & $\begin{array}{l}0,10 \mathrm{a} \\
0,10 \mathrm{a} \\
0,14 \mathrm{a} \\
0,06 \mathrm{a} \\
0,19 \mathrm{a}\end{array}$ & $\begin{array}{l}1,79 \mathrm{a} \\
1,38 \mathrm{a} \\
0,52 \mathrm{a} * \\
0,62 \mathrm{a} \\
0,93 \mathrm{a}\end{array}$ & $\begin{array}{l}0,57 \mathrm{a} \\
0,60 \mathrm{a} \\
0,64 \mathrm{a} \\
0,33 \mathrm{a} \\
1,16 \mathrm{a}\end{array}$ & $\begin{array}{l}2,54 \mathrm{~b} \\
2,34 \mathrm{~b} \\
4,26 \mathrm{a}^{*} \\
3,75 \mathrm{a}^{*} \\
3,38 \mathrm{ab}\end{array}$ & $\begin{array}{l}2,31 \mathrm{a} \\
2,62 \mathrm{a}^{*} \\
2,64 \mathrm{a}^{*} \\
2,03 \mathrm{a} \\
2,07 \mathrm{a}\end{array}$ \\
\hline \multirow[t]{2}{*}{$\begin{array}{l}\text { CV (\%) } \\
\text { Mata }\end{array}$} & $\begin{array}{c}4,4 \\
17,03\end{array}$ & $\begin{array}{c}3,6 \\
14,53\end{array}$ & $\begin{array}{r}15,5 \\
414,6\end{array}$ & $\begin{array}{r}21,4 \\
214,0\end{array}$ & $\begin{array}{c}62,4 \\
0,88\end{array}$ & $\begin{array}{c}95,2 \\
0,24\end{array}$ & $\begin{array}{c}73,4 \\
2,10\end{array}$ & $\begin{array}{c}114,0 \\
1,13\end{array}$ & $\begin{array}{c}16,3 \\
2,44\end{array}$ & $\begin{array}{c}21,6 \\
1,49\end{array}$ \\
\hline & \multicolumn{10}{|c|}{ Preparo convencional com a cultura do milho } \\
\hline $\begin{array}{l}\text { Pousio } \\
\text { Crotalária } \\
\text { Guandu } \\
\text { Mucuna } \\
\text { Sorgo }\end{array}$ & $\begin{array}{l}10,42 a^{*} \\
10,33 a^{*} \\
10,39 a^{*} \\
10,40 a^{*} \\
10,81 a^{*}\end{array}$ & $\begin{array}{l}9,01 a^{*} \\
8,53 a^{*} \\
8,59 a^{*} \\
8,62 a^{*} \\
8,79 a^{*}\end{array}$ & $\begin{array}{l}249,4 a \\
211,9 a^{*} \\
195,0 a^{*} \\
261,5 a \\
192,6 a^{*}\end{array}$ & $\begin{array}{l}163,8 \mathrm{a} \\
141,7 \mathrm{a} \\
138,5 \mathrm{a} \\
200,3 \mathrm{a} \\
171,1 \mathrm{a}\end{array}$ & $\begin{array}{l}0,47 \mathrm{a} \\
0,48 \mathrm{a} \\
0,51 \mathrm{a} \\
0,46 \mathrm{a} \\
0,47 \mathrm{a}\end{array}$ & $\begin{array}{l}0,28 \mathrm{a} \\
0,31 \mathrm{a} \\
0,30 \mathrm{a} \\
0,29 \mathrm{a} \\
0,29 \mathrm{a}\end{array}$ & $\begin{array}{l}2,38 \mathrm{a} \\
2,26 \mathrm{a} \\
3,33 \mathrm{a} \\
1,75 \mathrm{a} \\
2,45 \mathrm{a}\end{array}$ & $\begin{array}{l}1,83 \mathrm{a} \\
2,24 \mathrm{a} \\
2,33 \mathrm{a} \\
1,52 \mathrm{a} \\
1,87 \mathrm{a}\end{array}$ & $\begin{array}{l}2,38 \mathrm{a} \\
2,04 \mathrm{a} \\
1,84 \mathrm{a} \\
2,51 \mathrm{a} \\
1,81 \mathrm{a}\end{array}$ & $\begin{array}{l}1,82 \mathrm{a} \\
1,66 \mathrm{a} \\
1,61 \mathrm{a} \\
2,34 \mathrm{a} \\
1,98 \mathrm{a}\end{array}$ \\
\hline \multirow[t]{2}{*}{ Mata } & $\begin{array}{c}5,9 \\
17,03\end{array}$ & $\begin{array}{c}3,5 \\
14,53\end{array}$ & $\begin{array}{r}40,7 \\
414,6\end{array}$ & $\begin{array}{r}30,6 \\
214,0\end{array}$ & $\begin{array}{c}17,1 \\
0,88\end{array}$ & $\begin{array}{c}18,4 \\
0,24\end{array}$ & $\begin{array}{c}62,5 \\
2,10\end{array}$ & $\begin{array}{c}36,7 \\
1,13\end{array}$ & $\begin{array}{c}40,7 \\
2,44\end{array}$ & $\begin{array}{c}32,7 \\
1,49\end{array}$ \\
\hline & \multicolumn{10}{|c|}{ Semeadura direta com a cultura do milho } \\
\hline Pousio & $10,31 a^{*}$ & $8,87 a^{*}$ & $308,7 \mathrm{a}$ & $207,6 \mathrm{a}$ & $0,19 a^{*}$ & $0,32 \mathrm{a}$ & $0,62 a^{*}$ & $1,84 \mathrm{a}$ & $2,99 a$ & $2,35 \mathrm{a}$ \\
\hline Crotalária & $10,03 a^{*}$ & $8,48 a^{*}$ & $223,3 a^{*}$ & $199,4 \mathrm{a}$ & $0,25 a^{*}$ & $0,31 \mathrm{a}$ & $1,23 \mathrm{a}$ & $1,58 \mathrm{a}$ & $2,23 \mathrm{a}$ & $2,35 \mathrm{a}$ \\
\hline Guandu & $10,62 a^{*}$ & $9,01 a^{*}$ & $274,1 \mathrm{a}$ & $180,7 \mathrm{a}$ & $0,27 a^{*}$ & $0,16 \mathrm{a}$ & $1,04 \mathrm{a}$ & $0,94 \mathrm{a}$ & $2,55 \mathrm{a}$ & $2,00 \mathrm{a}$ \\
\hline Mucuna & $9,86 a^{*}$ & $8,55 a^{*}$ & $297,9 \mathrm{a}$ & $192,3 \mathrm{a}$ & $0,13 a^{*}$ & $0,36 \mathrm{a}$ & $0,44 a^{*}$ & $2,04 \mathrm{a}$ & $3,03 \mathrm{a}$ & $2,25 \mathrm{a}$ \\
\hline Sorgo & $9,93 a^{*}$ & $8,68 a^{*}$ & $251,3 \mathrm{a} *$ & $190,3 \mathrm{a}$ & $0,13 a *$ & $0,28 \mathrm{a}$ & $0,55 \mathrm{a}^{*}$ & $1,63 \mathrm{a}$ & $2,52 \mathrm{a}$ & $2,17 \mathrm{a}$ \\
\hline CV (\%) & 6,1 & 4,5 & 28,7 & 26,4 & 77,7 & 54,9 & 75,8 & 65,3 & 26,8 & 25,2 \\
\hline Mata & 17,03 & 14,53 & 414,6 & 214,0 & 0,88 & 0,24 & 2,10 & 1,13 & 2,44 & 1,49 \\
\hline
\end{tabular}

Médias nas colunas seguidas da mesma letra não diferem significativamente pelo teste de Tukey a $5 \%$, e as seguidas de * diferem significativamente da mata pelo teste de Dunnett a $5 \%$.

dois sistemas de preparo do solo é maior que a verificada em relação ao COT, indicando que o CBM, que representa a parte viva e mais ativa da matéria orgânica, é mais sensível ao preparo do solo que a parte morta, como também observaram Carneiro et al. (2009) e Pôrto et al. (2009).
O maior valor de CBM na mata é reflexo de uma situação particular para a microbiota do solo nesse sistema, que é estimulada pelo fornecimento contínuo de materiais orgânicos com diferentes graus de suscetibilidade à decomposição, originados da vegetação. A diversidade das espécies vegetais da mata 
nativa, notadamente maior que dos sistemas agrícolas avaliados, implica deposição na serapilheira de substratos orgânicos oxidáveis com composição variada. Além disso, existe maior diversidade de compostos orgânicos depositados na rizosfera, o que constitui fator favorável à sobrevivência e crescimento de diferentes grupos de microrganismos do solo. Nesse sentido, a abundância de microrganismos decompositores pode contribuir para estimular também a microfauna saprófita e predadora destes. Assim, as condições distintas do solo sob vegetação de mata, juntamente com a ausência de perturbações decorrentes de atividade antrópica, tornam possível a existência de maiores quantidades de CBM, indicando o maior equilíbrio da microbiota do solo nesse ecossistema (D’Andréa, 2002; Perez et al., 2004; Pôrto et al., 2009).

A maior redução em CBM nos experimentos sob preparo convencional sinaliza que esse sistema de preparo contribui para reduzir a quantidade e, possivelmente, a diversidade de microrganismos, com grande impacto na microbiota do solo. É possível que as adições de $\mathrm{C}$ oxidável nesse sistema não estejam sendo suficientes para atender à demanda para a manutenção da biomassa existente, o que ocasiona maior decréscimo nos valores de CBM. Silva et al. (2007) verificaram que a semeadura direta propiciou maiores valores de CBM em relação ao preparo convencional. Esse fato tem sido atribuído ao acúmulo de resíduos culturais e, por conseguinte, do C orgânico total. Maior quantidade de CBM reflete a presença de maior quantidade de matéria orgânica ativa no solo, capaz de manter elevada a taxa de decomposição de restos vegetais e, portanto, de reciclar mais nutrientes (Stenberg, 1999).

Nos experimentos com o milho, houve correlação significativa e positiva entre o COT e CBM ( $r=0,72$, $\mathrm{p}<0,05$, e r $=0,84, \mathrm{p}<0,01$, respectivamente para o preparo convencional e a semeadura direta). Nos experimentos com o feijoeiro, a correlação foi significativa apenas para a semeadura direta $(r=0,74$, p < 0,05). Conceição et al. (2005) e Pôrto et al. (2009) também observaram correlação significativa entre esses dois atributos, demonstrando que a quantidade de substrato orgânico disponível no sistema tem efeito na quantidade de CBM. Entretanto, considerando que nos experimentos com o feijoeiro as plantas de cobertura diferiram quanto ao seu efeito no CBM e não diferiram quanto ao seu efeito no COT, infere-se que a composição do substrato orgânico, afetando a sobrevivência e o crescimento de diferentes grupos de microrganismos do solo, também é importante.

\section{Respiração basal do solo (RBS)}

Não foram observadas diferenças significativas entre as plantas de cobertura quanto à RBS, tanto na camada de 0,00-0,10 $\mathrm{m}$ como na de 0,10-0,20 m, seja sob preparo convencional (Quadro 1) ou semeadura direta (Quadro 1), nos experimentos com as culturas do feijoeiro e milho.
Nos experimentos com semeadura direta, a RBS na camada superficial do solo foi menor sob as plantas de cobertura em comparação com a mata, o que não se verificou nos experimentos sob preparo convencional, em que não houve diferença significativa entre as plantas de cobertura e a mata (Quadro 1). Na camada de 0,10-0,20 m, as plantas de cobertura não diferiram da mata quanto à RBS, em nenhum dos experimentos. D’Andréa et al. (2002) não encontraram diferenças significativas nos valores de RBS entre Cerrado nativo, pastagem e sistemas agrícolas sob preparo convencional e semeadura direta. Silva et al. (2007) não verificaram diferenças entre preparo convencional e semeadura direta quanto a esse atributo biológico do solo. Pôrto et al. (2009), por sua vez, verificaram maior valor de RBS na mata que em sistemas de sucessão e de consorciação de cultivos, e Jakelaitis et al. (2008) e Carneiro et al. (2009) observaram maiores valores na mata do que em áreas de pastagem e de semeadura direta de milho.

A maior liberação de $\mathrm{CO}_{2}$ geralmente está associada à maior atividade biológica, que, por sua vez, está relacionada diretamente com a quantidade de C lábil no solo. Entretanto, a interpretação dos resultados da atividade biológica deve ser feita com critério, uma vez que elevados valores de respiração nem sempre indicam condições desejáveis: a curto prazo pode significar liberação de nutrientes para as plantas e, a longo prazo, perda de C orgânico do solo para a atmosfera (Parkin et al., 1996). Dessa forma, elevados valores de RBS podem indicar tanto situações de distúrbio quanto de alto nível de produtividade do sistema (Islam \& Weil, 2000a).

Nos experimentos com o feijoeiro, houve correlação significativa e positiva entre COT e RBS ( $r=0,83$, $\mathrm{p}<0,01$, e $\mathrm{r}=0,76, \mathrm{p}<0,05$, respectivamente para $\mathrm{o}$ preparo convencional e a semeadura direta). Nos experimentos com o milho, a correlação foi significativa apenas para o preparo convencional $(\mathrm{r}=$ 0,95, p < 0,01). Pôrto et al. (2009) também observaram correlação significativa entre esses dois atributos. Araújo et al. (2007) verificaram que, apesar de a atividade da biota do solo ser limitada pela quantidade de substrato carbonado, não houve correlação da RBS com o CBM e sim entre RBS e matéria orgânica do solo, demonstrando que, independentemente dos teores de $\mathrm{C}$ da biomassa microbiana, existe relação estreita entre a matéria orgânica e a atividade dos microrganismos do solo. Corroborando os resultados destes autores, também não foi verificada correlação significativa entre RBS e CBM no presente trabalho.

\section{Quociente metabólico $\left(q \mathrm{CO}_{2}\right)$}

Nos experimentos com as culturas do feijoeiro e do milho não foram observadas diferenças significativas entre as plantas de cobertura quanto ao $q \mathrm{CO}_{2}$, tanto na camada de 0,00-0,10 m como na de 0,10-0,20 m, seja sob preparo convencional ou semeadura direta (Quadro 1). Houve diferenças significativas entre as 
plantas de cobertura e a mata apenas na camada superficial do solo sob semeadura direta, onde o guandu, no experimento com o feijoeiro (Quadro 1), e o pousio, a mucuna e o sorgo, com o milho (Quadro 1), propiciaram menores valores de $q \mathrm{CO}_{2}$ que a mata.

Tomando a mata como referência, observou-se aumento nos valores médios de $q \mathrm{CO}_{2}$ na camada superficial do solo da ordem de 46,8 e 15,9\% para os experimentos sob preparo convencional, e redução de 50,1 e $63,0 \%$ na semeadura direta, respectivamente com as culturas do feijoeiro e do milho.

Valores elevados de $q \mathrm{CO}_{2}$ são indicativos de ecossistemas submetidos a alguma condição de estresse ou distúrbio (Moreira \& Siqueira, 2006), que é o caso das parcelas submetidas ao preparo convencional do solo. Esse preparo promove rompimento dos macro e microagregados e, consequentemente, torna a matéria orgânica mais suscetível ao ataque microbiano, o que aumenta a taxa de mineralização e liberação de $\mathrm{CO}_{2}$ para a atmosfera (Six et al., 2000). Silva et al. (2007) observaram maior $q \mathrm{CO}_{2}$ no solo sob preparo convencional do que em semeadura direta. D’Andréa et al. (2002), por sua vez, não encontraram diferenças significativas nos valores de $q \mathrm{CO}_{2}$ entre Cerrado nativo, pastagem e sistemas agrícolas sob preparo convencional e semeadura direta. Jakelaitis et al. (2008) e Pôrto et al. (2009), entretanto, constataram menor valor de $q \mathrm{CO}_{2}$ no solo sob mata do que no solo sob outros sistemas de uso.

À medida que a biomassa microbiana se torna mais eficiente na utilização de recursos do ecossistema, menos $\mathrm{CO}_{2}$ é perdido pela respiração e maior proporção de $\mathrm{C}$ é incorporada aos tecidos microbianos, o que resulta em diminuição do $q \mathrm{CO}_{2}$. Menores valores de $q \mathrm{CO}_{2}$ indicam agroecossistemas mais estáveis, e a substituição da vegetação nativa acelera a decomposição de resíduos, com aumento do valor de $q \mathrm{CO}_{2}$ (Silva et al., 2007).

\section{Quociente microbiano ( $q$ MIC)}

O qMIC, em condições normais, varia de 1 a $4 \%$, e valores inferiores a $1 \%$ podem ser atribuídos a algum fator limitante à atividade da biomassa microbiana (Jakelaitis et al., 2008). Neste trabalho, em todos os experimentos, o valor de $q \mathrm{MIC}$ foi superior a $1 \%$ (Quadro 1), indicando que o sistema de produção orgânico favoreceu a atividade dos microrganismos, independentemente do preparo do solo e das culturas de cobertura.

Foram observadas diferenças significativas entre as plantas de cobertura, quanto ao $q \mathrm{MIC}$, apenas na camada de 0,00-0,10 $\mathrm{m}$ dos experimentos com o feijoeiro (Quadro 1). No experimento sob preparo convencional (Quadro 1), o guandu propiciou maiores valores desse atributo em relação às demais plantas de cobertura, com exceção da mucuna. Sob semeadura direta (Quadro 1), o qMIC foi maior sob guandu e mucuna em relação à crotalária e ao pousio. Esses resultados refletem o efeito dessas plantas de cobertura sob o CBM, uma vez que elas não diferiram quanto ao COT.

Considerando a camada superficial dos experimentos com o feijoeiro, o guandu, sob preparo convencional (Quadro 1) e semeadura direta, e a mucuna, também sob semeadura direta (Quadro 1), propiciaram maiores valores de $q \mathrm{MIC}$ em relação à mata. Na camada de 0,10-0,20 m isso ocorreu para todas as plantas de cobertura, sob preparo convencional (Quadro 1), e para o guandu e a crotalária, sob semeadura direta (Quadro 1). Nos experimentos com o milho (Quadro 1), as plantas de cobertura não diferiram da mata quanto a esse atributo. D'Andréa et al. (2002) e Silva et al. (2007) encontraram maiores valores de $q \mathrm{MIC}$ em solo de mata nativa em comparação com os sistemas agrícolas. Entretanto, Pôrto et al. (2009) não observaram diferenças entre mata e diferentes sistemas de uso do solo com relação a esse atributo, e Jakelaitis et al. (2008) não verificaram diferenças entre mata e pastagem formada pelo consórcio de milho com Brachiaria brizantha ou pastagem de $B$. brizantha após rotação pastagem-soja-pastagem. Esses últimos autores, contudo, observaram menor valor de $q \mathrm{MIC}$ em área cultivada com milho em semeadura direta.

$\mathrm{O}$ menor valor de $q \mathrm{MIC}$ no solo sob mata reflete a menor utilização de $\mathrm{C}$ pela microbiota do solo, podendo estar associado à acidez do solo ou a fatores como limitação de nutrientes, ou mesmo à qualidade da matéria orgânica aportada em relação à das plantas de cobertura.

Tomando a mata como referência, observou-se aumento nos valores médios de $q \mathrm{MIC}$ na camada superficial do solo na ordem de 33,4 e 9,2 \% para os experimentos sob semeadura direta e redução de 3,5 e 13,6 \% para os experimentos sob preparo convencional, respectivamente com as culturas do feijoeiro e do milho. A tendência de maiores valores de $q \mathrm{MIC}$ na semeadura direta concorda com os resultados de Balota et al. (1998) e Silva et al. (2007). Esse quociente é influenciado por diversos fatores, como o grau de estabilização do C orgânico e o histórico de manejo do solo.

As variações em $q \mathrm{MIC}$ refletem o padrão do aporte de matéria orgânica do solo, a eficiência da conversão do $\mathrm{C}$ microbiano, as perdas de $\mathrm{C}$ do solo e a estabilização do C orgânico pelas frações minerais do solo. Assim, $q \mathrm{MIC}$ indica se o conteúdo de $\mathrm{C}$ está se mantendo estável ou variando de acordo com as condições impostas ao sistema (Tótola \& Chaer, 2002). Entretanto, segundo Pôrto et al. (2009), alguns autores têm demonstrado que esse atributo, da forma como vem sendo determinado, relação entre CBM total e COT, pode apresentar limitações para predizer a qualidade do solo em determinadas situações. Islam \& Weil (2000a) verificaram que o emprego de CBM ativa, obtido pela técnica da respiração induzida, em vez do CBM total na determinação do $q \mathrm{MIC}$ mostrouse mais eficiente para predizer as alterações provenientes de práticas de manejo. 


\section{CONCLUSÕES}

1. As principais alterações nos atributos biológicos do solo, com o uso agrícola, ocorreram na camada superficial, em que, de maneira geral, os valores de C da biomassa microbiana foram menores que no solo sob mata, sendo esse fato mais pronunciado nas áreas sob preparo convencional.

2. O quociente metabólico mostrou-se sensível às alterações decorrentes do preparo do solo, apresentando valores mais favoráveis na camada superficial do solo sob semeadura direta.

\section{LITERATURA CITADA}

ALBUQUERQUE, J.A.; ARGENTON, J.; BAYER, C.; WILDNER, L.P. \& KUNTZE, M.A.G. Relação de atributos do solo com a agregação de um Latossolo Vermelho sob sistemas de preparo e plantas de verão para cobertura do solo. R. Bras. Ci. Solo, 29:415-424, 2005.

ALMEIDA, V.P.; ALVES, M.C.; SILVA, E.C. \& OLIVEIRA, S.A. Rotação de culturas e propriedades físicas e químicas em Latossolo Vermelho de cerrado sob preparo convencional e semeadura direta em adoção. R. Bras. Ci. Solo, 32:1227. $1237,2008$.

ALVAREZ, R.; DOAZ, R.A.; BARBERO, N.; SANTANATOGLIA, O.J. \& BLOTTA, L. Soil organic carbon, microbial biomass and $\mathrm{CO}_{2}$ C production from three tillage system. Soil Till. Res., 33:17-28, 1995.

AMADO, T.J.C.; BAYER, C.; ELTZ, F.L.F. \& BRUM, A.C.R Potencial de culturas de cobertura em acumular carbono e nitrogênio no solo no plantio direto e a melhoria da qualidade ambiental. R. Bras. Ci. Solo, 25:189-197, 2001.

ANDERSON, J.P.E. \& DOMSCH, K.H. The metabolic quotient $\left(q \mathrm{CO}_{2}\right)$ as a specific activity parameter to asses the effects of environmental conditions, such as $\mathrm{pH}$, on the microbial biomass of forest soils. Soil Biol. Biochem., 25:393 $395,1993$.

ARAÚJO, R.; GOEDERT, W.J. \& LACERDA, M.P.C. Qualidade de um solo sob diferentes usos e sob cerrado nativo. $R$. Bras. Ci. Solo, 31:1099-1108, 2007.

BALOTA, E.L.; COLOZZI-FILHO, A.; ANDRADE, D.S. \& HUNGRIA, M. Biomassa microbiana e sua atividade em solos sob diferentes sistemas de preparo e sucessão de culturas. R. Bras. Ci. Solo, 22:641-649, 1998.

BREMER, E. \& ELLERT, K. Soil quality indicators: A review with implications for agricultural ecosystems in Alberta. Edmonton, Alberta Agriculture, Food and Rural Development, 2004. 38p.

CARNEIRO, M.A.C.; SOUZA, E.D.; REIS, E.F.; PEREIRA, H.S \& AZEVEDO, W.R. Atributos físicos, químicos e biológicos de solo de Cerrado sob diferentes sistemas de uso e manejo. R. Bras. Ci. Solo, 33:147-157, 2009.
CONCEIÇÃO, P.C.; AMADO, T.J.C.; MIELNICZUK, J. \& SPAGNOLLO, E. Qualidade do solo em sistemas de manejo avaliada pela dinâmica da matéria orgânica e atributos relacionados. R. Bras. Ci. Solo, 29:777-788, 2005.

COSTA, E.A.; GOEDERT, W.J. \& SOUSA, D.M.G. Qualidade de solo submetido a sistemas de cultivo com preparo convencional e plantio direto. Pesq. Agropec. Bras., 41:1185-1191, 2006.

CUNHA, E.Q.; STONE, L.F.; MOREIRA, J.A.A.; FERREIRA, E.P.B.; DIDONET, A.D. \& LEANDRO, W.M. Sistemas de preparo do solo e culturas de cobertura na produção orgânica de feijão e milho. I - Atributos físicos do solo. R. Bras. Ci. Solo, 35:589-602, 2011.

D'ANDREA, A.F.; SILVA, M.L.N.; CURI, N.; SIQUEIRA, J.O. \& CARNEIRO, M.A.C. Atributos biológicos indicadores da qualidade do solo em sistemas de manejo na Região do Cerrado do Sul do Estado de Goiás. R. Bras. Ci. Solo, 26:913-923, 2002

EMPRESA BRASILEIRA DE PESQUISA AGROPECUÁRIA EMBRAPA. Manual de métodos de análise de solo. 2.ed. Rio de Janeiro, 1997. 212p. (Embrapa- CNPS. Documentos, 1)

EMPRESA BRASILEIRA DE PESQUISA AGROPECUÁRIA EMBRAPA. Sistema brasileiro de classificação de solo. 2.ed. Rio de Janeiro, 2006. 306p.

ISLAM, K.R. \& WEIL, R.R. Land use effects on soil quality in a tropical forest ecosystem of Bangladesh. Agric. Ecosyst. Environ., 79:9-16, 2000a.

ISLAM, K.R. \& WEIL, R.R. Soil quality indicator properties in mid-Atlantic soils as influenced by conservation management. J. Soil Water Conserv., 55:69-79, 2000 b.

JAKELAITIS, A.; SILVA, A.A.; SANTOS, J.B. \& VIVIAN, R. Qualidade da camada superficial de solo sob mata, pastagens e áreas cultivadas. Pesq. Agropec. Trop., 38:118127,2008

JENKINSON, D.S. \& POWLSON, D.S. The effects of biocidal treatments on metabolism in soil. A method for measuring soil biomass. Soil Biol. Biochem., 8:209-213, 1976.

MENDES, I.C.; SOUZA, L.V.; RESCK, D.V.S. \& GOMES, A.C. Propriedades biológicas em agregados de um Latossolo Vermelho-Escuro sob plantio convencional e direto no Cerrado. R. Bras. Ci. Solo, 27:435-443, 2003.

MOREIRA, F.M.S. \& SIQUEIRA, J.O. Microbiologia e bioquímica do solo. 2.ed. Lavras, Universidade Federal de Lavras, 2006. 729p.

NASCIMENTO, J.T.; SILVA, I.F.; SANTIAGO, R.D. \& SILVA NETO, L.F. Efeito de leguminosas nos atributos físicos e carbono orgânico de um Luvissolo. R. Bras. Ci. Solo, 29:825-831, 2005

PARKIN, T.B.; DORAN, J.W. \& FRANCOP-VIZCAINO, E. Field and laboratory tests of soil respiration. In: DORAN, J.W. \& JONES, A., eds. Method for assessing soil quality. Madison, Soil Science Society of America, 1996. p.231245. 
PEREZ, K.S.; RAMOS, M.L.G. \& McMANUS, C. Nitrogênio da biomassa microbiana em solo cultivado com soja, sob diferentes sistemas de manejo, nos Cerrados. Pesq. Agropec. Bras., 40:137-144, 2004.

PÔRTO, M.L.; ALVES, J.C.; DINIZ, A.A.; SOUZA, A.P. \& SANTOS, D. Indicadores biológicos de qualidade do solo em diferentes sistemas de uso no Brejo Paraibano. Ci. Agrotec., 33:1011-1017, 2009.

SANTOS, G.A. \& CAMARGO, F.A.O. Fundamentos da matéria orgânica do solo: Ecossistemas tropicais e subtropicais. Porto Alegre, Gênesis, 1999. 508p.

SANTOS, T.E.B.; NAKAYAMA, F.T.; ARF, O. \& CASSIOLATO, A.M.R. Variáveis microbiológicas e produtividade do arroz sob diferentes manejos do solo e água. Acta Sci. Agron., 29:355-366, 2007.

SAS Institute Inc. SAS/STAT procedure guide for personal computers: version 5. Cary, 1999.

SILVA, I.R. \& MENDONÇA, E.S. Matéria orgânica do solo. In: NOVAIS, G.N.; ALVAREZ V., V.H.; BARROS, N.F.; FONTES, R.L.F.; CANTARUTTI, B. \& NEVES, J.C.L., eds. Fertilidade do solo. Viçosa, MG, Sociedade Brasileira de Ciência do Solo, 2007. p.275-374.

SILVA, M.B.; KLIEMANN, H.J.; SILVEIRA, P.M. \& LANNA, A.C. Atributos biológicos do solo sob influência da cobertura vegetal e do sistema de manejo. Pesq. Agropec. Bras., 42:1755-1761, 2007.

SIX, J.; PAUSTIAN, K.; ELLIOT, E.T. \& COMBRINK, C. Soil structure and organic matter: Distribution of aggregatesize classes and aggregate associated carbon. Soil Sci. Soc. Am. J., 64:681-689, 2000.
SOUSA NETO, E.L.; ANDRIOLI, I.; BEUTLER, A.N. \& CENTURION, J.F. Atributos físicos do solo e produtividade de milho em resposta a culturas de pré-safra. Pesq. Agropec. Bras., 43:255-260, 2008.

SOUSA-SILVA, J.C. \& CAMARGO, A.J.A. A flora e a fauna do Cerrado. In: ALBUQUERQUE, A.C.S. \& SILVA, A.G., eds. Agricultura tropical: Quatro décadas de inovações tecnológicas, institucionais e políticas. Brasília, Embrapa Informação Tecnológica, 2008. v.2. p.149-201.

SOUZA, L.M.; CASTILHOS, D.D.; MORSELLI, T.B.G.A. \& CASTILHOS, R.M.V. Influência da aplicação de diferentes vermicompostos na biomassa microbiana do solo após cultivo de alface. R. Bras. Agroci., 12:429-434, 2006.

STENBERG, B. Monitoring soil quality of arable land: Microbiological indicators. Acta Agric. Scand., Sect. B Soil Plant Sci., 49:1-24, 1999.

TÓTOLA, M.R. \& CHAER, G.M. Microorganismos e processos microbiológicos como indicadores de qualidade dos solos. In: ALVAREZ V., V.H.; SCHAEFER, C.E.G.R.; BARROS, N.F.; MELLO, J.W.V. \& COSTA, L.M., eds. Tópicos em ciência do solo. Viçosa, MG, Sociedade Brasileira de Ciência do Solo, 2002. v.2. p.195-276.

VANCE, E.D.; BROOKES, P.C. \& JENKINSON, D.S. An extraction method for measuring soil microbial biomass C. Soil Biol. Biochem., 19:703-707, 1987.

VASCONCELLOS, C.A.; CAMPOLINA, D.C.A.; SANTOS, F.G.; EXEL PITTA, G.V. \& MARRIEL, I.E. Resposta da soja e da biomassa de carbono do solo aos resíduos de cinco genótipos de sorgo. R. Bras. Ci. Solo, 23:69-77, 1999. 
